\title{
THz Electro-absorption Effect in Quantum Dots
}

Turchinovich, Dmitry; Monozon, Boris S.; Livshits, Daniil A.; Rafailov, Edik U.; Hoffmann, Matthias C.

\section{Published in:}

Proceedings of EDISON 17

Publication date:

2011

Link back to DTU Orbit

Citation (APA):

Turchinovich, D., Monozon, B. S., Livshits, D. A., Rafailov, E. U., \& Hoffmann, M. C. (2011). THz Electroabsorption Effect in Quantum Dots. In Proceedings of EDISON $17 \mathrm{http}: / /$ www.asu.edu/aine/EDISON17/

\section{General rights}

Copyright and moral rights for the publications made accessible in the public portal are retained by the authors and/or other copyright owners and it is a condition of accessing publications that users recognise and abide by the legal requirements associated with these rights.

- Users may download and print one copy of any publication from the public portal for the purpose of private study or research.

- You may not further distribute the material or use it for any profit-making activity or commercial gain

- You may freely distribute the URL identifying the publication in the public portal

If you believe that this document breaches copyright please contact us providing details, and we will remove access to the work immediately and investigate your claim 


\title{
$\mathrm{THz}$ Electro-absorption Effect in Quantum Dots
}

\author{
Dmitry Turchinovich ${ }^{1}$, Boris S. Monozon ${ }^{2}$, Daniil A. Livshits ${ }^{3}$, Edik U. Rafailov ${ }^{4}$, \\ and Matthias C. Hoffmann ${ }^{5}$ \\ ${ }^{1}$ DTU Fotonik - Department of Photonics Engineering, Technical University of Denmark, \\ Ørsteds Plads 343, DK-2800 Kgs. Lyngby, Denmark \\ email: dmtu@fotonik.dtu.dk, phone: + 45 4525 3653, fax:+ 4545256581 \\ ${ }^{2}$ Department of Physics, State Marine Technical University, Lotsmanskaya 3, 19008 St.Petersburg, Russia \\ ${ }^{3}$ Innolume GmbH, Konrad-Adenauer-Allee 11, 44263, Dortmund, Germany \\ ${ }^{4}$ School of Engineering, Physics and Mathematics, University of Dundee, Dundee DD1 4HN, UK \\ ${ }^{5}$ Max Planck Research Department for Structural Dynamics, CFEL, University of Hamburg, \\ DESY, Notkestr. 85, 22607 Hamburg, Germany
}

In a $\mathrm{THz}$ pump - optical probe experiment we demonstrate an instantaneous electro-absorption effect in InGaAs/GaAs quantum dots, induced by the electric field of a single-cycle $\mathrm{THz}$ pulse with $3 \mathrm{THz}$ bandwidth and with free-space peak electric field reaching $220 \mathrm{kV} / \mathrm{cm}$. The transient modulation of QD ground state optical absorption at $1040 \mathrm{~nm}$ coherently follows the evolution of the absolute value of $\mathrm{THz}$ electric field. The optical modulation signal was found to be as short as 460 fs at FWHM, and retained the $3 \mathrm{THz}$ bandwidth of the THz pulse. Optical absorption modulation in QDs by the THz field is dominated by absorption quenching effect due to the electron- and hole wavefunction separation in $\mathrm{THz}$ field, rather than by the Stark shift away from the optical probe.

We have performed a THz pump - optical probe experiment on InGaAs/GaAs quantum dot (QD) sample [1]. The sample used in our experiments was a QD-based semiconductor saturable absorber mirror (SESAM), comprising of a broad-band $\mathrm{GaAs} / \mathrm{Al}_{0.9} \mathrm{Ga}_{0.1} \mathrm{As}$ distributed Bragg reflector (DBR), and a QD absorber layer featuring 80 layers of sub-monolayer - grown $\mathrm{In}_{0.5} \mathrm{Ga}_{0.5} \mathrm{As} / \mathrm{GaAs} \mathrm{QDs}$, alternated by the GaAs barriers of 10-14 nm thickness. The QD ground state resonance was at $1040 \mathrm{~nm}$, providing the strong dip in SESAM reflectivity at this wavelength (see Fig. 1). The sample was similar to the one studied in Ref. [2]. A

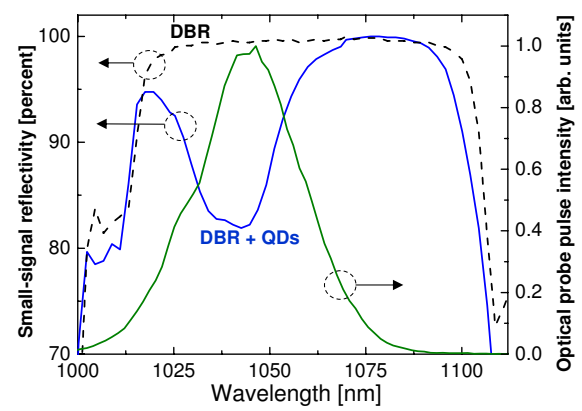

FIG. 1: Small-signal reflectivity spectrum of the whole QD sample (solid line), a bare DBR (dashed line), and the intensity spectrum of the probe laser pulse at QD ground state resonance at $1040 \mathrm{~nm}$.

strong THz pump pulse with peak electric field in air of $220 \mathrm{kV} / \mathrm{cm}$ and a bandwidth of $3 \mathrm{THz}$ (Fig. 2(a)) was produced by tilted pulse front optical rectification of an $800 \mathrm{~nm}, 80-\mathrm{fs}$ Ti:Sa amplifier pulse in a lithium niobate crystal [3]. A weak probe pulse at $1040 \mathrm{~nm}$, resonant with a ground state QD absorption in the absence of $\mathrm{THz}$ field, was produced by an OPA synchronized to the THz source. Our main result - the change in optical probe reflectivity $\Delta R / R$ of a QD SESAM sample, induced by
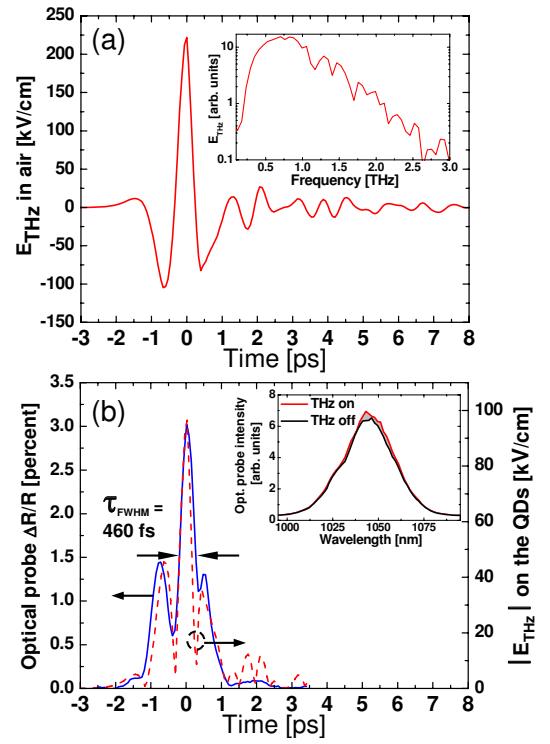

FIG. 2: (a) THz pulse with peak free-space electric field strength of $220 \mathrm{kV} / \mathrm{cm}$. Inset: its amplitude frequency spectrum. (b) Our main result: Solid line - reflectivity modulation $\Delta R / R$ of the probe signal at $1040 \mathrm{~nm}$ in the QD sample, under influence of $\mathrm{THz}$ pulse from (a). Dashed line - absolute value of the electric field in the $\mathrm{THz}$ pulse from (a) experienced by QDs. Inset: spectrum of the optical probe signal with and without peak electric field of the $\mathrm{THz}$ signal from (a) on the QD sample.

the THz pulse, as a function of time is shown in Fig. 2(b) together with the absolute value of the electric field on the QDs. The latter was calculated taking into account the $\mathrm{THz}$ field transmission coefficient at the sample GaAs interface $t_{T H z}=0.435$. The values of $\Delta R / R$ are always positive, demonstrating that resonant optical absorption in QDs always decreases in the presence of a $\mathrm{THz}$ field.

The electric field of the $\mathrm{THz}$ pump pulse induces a quantum-confined Stark effect (QCSE) [4] on the QDs, 

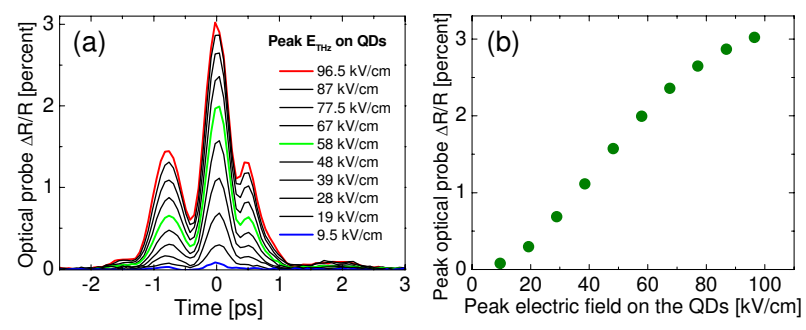

FIG. 3: (a) Reflectivity modulation $\Delta R / R$ of the optical probe in the QD sample, at various $\mathrm{THz}$ peak electric field strength. (b) Peak values of $\Delta R / R$ of the signals from (a), as a function of peak $\mathrm{THz}$ electric field on the QDs.

which manifests itself in two ways: (i) in decrease of optical transition energy (Stark shift) as a result of band structure tilt, moving the QD absorption out of the optical probe spectrum, and (ii) in a reduced wavefunctions overlap in the presence of electric field $M(E)=\left\langle\psi_{e} \mid \psi_{h}\right\rangle$. The value of $|M(E)|^{2}$ dictates the strength of optical absorption in QDs. The Stark shift and absorption quenching may be dominant in different spectral parts of the absorption peak [5]. In the inset of Fig. 2(b) the optical spectra of probe pulses are shown in the case when optical probe pulse was in temporal overlap with the peak of the $\mathrm{THz}$ pulse from Fig. 2(a), and when the $\mathrm{THz}$ pulse was blocked. The $\mathrm{THz}$ field - induced modulation of optical probe pulse is distributed more or less spectrally homogenously, which suggests that the absorption quenching is likely to be the dominating effect here.

Controllable attenuation of the $\mathrm{THz}$ electric field by a pair of wire-grid polarizers [6] allowed us to observe the nonlinear dependency of the electro-absorption modulation depth on the peak $\mathrm{THz}$ electric field, typical for QCSE mostly dominated by quenching contribution [4] - see Fig. 3. As expected from the 3D geometry of the $\mathrm{QD}$, we found no dependency of the electro-absorption modulation strength on the polarization of $\mathrm{THz}$ signal. We observed a weak dependency on the polarization of the optical probe signal, which is most likely due to a well-known optical absorption anisotropy in the QDs [7].

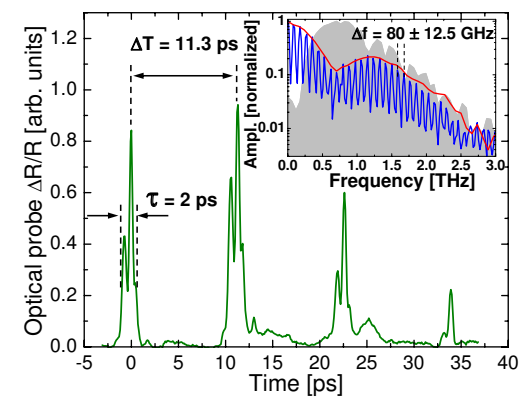

FIG. 4: $\Delta R / R$ of the optical probe, resulting from multiple reflections of a single $\mathrm{THz}$ pulse in the $\mathrm{QD}$ sample. Inset: Amplitude Fourier spectra of the isolated first $\Delta R / R$ pulse around 0 ps (red line), and of the full multi-pulse sequence (blue line). Amplitude Fourier spectrum of a single THz pulse is shown in the background.
We note that in Refs. [8, 9] an electro-absorption modulation was also observed at the exciton resonance in quantum wells (QWs) and carbon nanotubes (CNTs). In [8] the QWs were polarized in the plane by the strong $\mathrm{THz}$ pulse, and increase of $\mathrm{THz}$ field strength lead to exciton ionization. In [9] the CNTs were polarized along the long dimension, and the modulation speed limit of around $1 \mathrm{THz}$ was assigned to exciton dephasing.

In Fig. 4 we demonstrate an optical modulation of QDs induced by a train of $\mathrm{THz}$ pulses, originating from the multiple reflection of a single $\mathrm{THz}$ pulse between the surface of the sample, and the metal mirror attached to the back side of its $450 \pm 25 \mu \mathrm{m}$ - thick GaAs substrate. This experiment emulates the direct THz-to-optical encoding in a ultra-high-speed THz-frequency digital communication channel [10]. The inter-pulse interval of 11.3 ps, i.e. the repetition rate of $88 \mathrm{GHz}$, results in the data rate of $88 \mathrm{Gbit} / \mathrm{s}$. The ratio of inter-pulse interval to the individual pulse duration $11.3 \mathrm{ps} / 2 \mathrm{ps}=5.65$ shows the achievable wireless data capacity of at least $88 \mathrm{Gbit} / \mathrm{s} \times$ $5.65=0.5 \mathrm{Tbit} / \mathrm{s}$ using this modulation scheme.

In the inset of Fig. 4 the amplitude spectra of the isolated $\Delta R / R$ pulse, and of the whole multi-pulse sequence are shown, with the amplitude spectrum of the single $\mathrm{THz}$ pulse in the background. The full bandwidth of the $\mathrm{THz}$ pulse of $3 \mathrm{THz}$ is encoded onto the optical probe signal, demonstrating the coherency of electro-absorption modulation in QDs with the driving $\mathrm{THz}$ field. This coherency will be most likely limited by the $\mathrm{THz}$ frequencies high enough to excite intra-band transitions in the QDs, such as approximately $10 \mathrm{THz}$ or higher.

We acknowledge the financial support from Danish Proof-of-Concept Foundation (Grant 7.7 Ultra-highspeed wireless data communications), EU FP7 program (FAST-DOT, Grant 224338), and Max Planck Society.

[1] M. C. Hoffmann, B. S. Monozon, D. Livshits, E. U. Rafailov, and D. Turchinovich, Appl. Phys. Lett. 97, 231108 (2010).

[2] H. P. Porte, P. U. Jepsen, N. Daghestani, E. U. Rafailov, and D. Turchinovich, Appl. Phys. Lett. 94, 262104 (2009).

[3] K.-L. Yeh, M. C. Hoffmann, J. Hebling, and K. A. Nelson, Appl. Phys. Lett. 90, 171121 (2007).

[4] D. A. B. Miller, D. S. Chemla, T. C. Damen, A. C. Gossard, W. Wiegmann, T. H. Wood, and C. A. Burrus, Phys. Rev. Lett. 53, 2173-2176 (1984).

[5] X. Liu, E. U. Rafailov, D. Livshits, and D. Turchinovich, Appl. Phys. Lett. 97, 051103 (2010).

[6] M. C. Hoffmann and D. Turchinovich, Appl. Phys. Lett. 96, 151110 (2010).

[7] S. Cortez, O. Krebs, P. Voisin, and J. M. Gérard, Phys. Rev. B 63, 233306 (2001).

[8] H. Hirori, M. Nagai, K. Tanaka, Phys. Rev. B 81, 081305(R) (2010).

[9] T. Ogawa, S. Watanabe, N. Minami, and R. Shimano, Appl. Phys. Lett. 97, 041111 (2010).

[10] D. Turchinovich, patent WO2011/015200 